Voix et Images

\title{
Un scénario motif dans le champ des best-sellers
}

\section{Jacques Lemieux et Denis Saint-Jacques}

Volume 15, numéro 2 (44), hiver 1990

Pratiques illicites

URI : https://id.erudit.org/iderudit/200839ar

DOI : https://doi.org/10.7202/200839ar

Aller au sommaire du numéro

Éditeur(s)

Université du Québec à Montréal

ISSN

0318-9201 (imprimé)

1705-933X (numérique)

Découvrir la revue

Citer cet article

Lemieux, J. \& Saint-Jacques, D. (1990). Un scénario motif dans le champ des best-sellers. Voix et Images, 15(2), 260-268. https://doi.org/10.7202/200839ar d'utilisation que vous pouvez consulter en ligne.

https://apropos.erudit.org/fr/usagers/politique-dutilisation/ 


\title{
Un scénario motif dans le champ des best-sellers *
}

\author{
Jacques Lemieux, université Laval \\ et Denis Saint-Jacques, université Laval
}

Nous voulons examiner ici les structures narratives d'un certain nombre de best-sellers récents à la lumière des apports théoriques et méthodologiques du Lector in Fabula d'Umberto Eco ${ }^{1}$. Nous nous intéresserons particulièrement à la question des inférences de scénarios communs et intertextuels ${ }^{2}$, aux topics ${ }^{3}$ et au lecteur modèle 4 . Les ouvrages qui fournissent le corpus d'expérimentation sont ceux que nous avons étudiés dans une recherche portant sur le phénomène des best-sellers au Québec de 1970 à $1982^{5}$. Nous fournissons en annexe pour les besoins de cet article une liste restreinte de 15 titres représentatifs des plus grands succès de l'époque sur le marché québécois; on signalera que ces livres forment une sélection orientée en fonction du thème de cette étude et non un ensemble statistiquement typique. On trouvera une discussion de la constitution de ce corpus dans "Ce que racontent les best-sellers" 6 .

Nos travaux sur les best-sellers se sont développés dans une double perspective socio-économique et narratologique: d'une part, analyse de la production et de la distribution, et de l'autre, étude textuelle des récits les plus représentatifs. C'est de ce second volet qu'il sera question ici. Ayant volontairement écarté du corpus de notre recherche les récits de série (policiers, espionnage, science-fiction, sentimental, etc.) dans le souci de traiter les succès les plus importants et les

Cet article présente une version remaniée d'une communication faite au congrès des Sociétés savantes de 1987, à l'APFUCC, dans un atelier consacré à la narratologie, sous la direction de Julia Bettinotti de l'Université du Québec à Montréal.

1 Umberto Eco, Lector in fabula ou la Coopération interprétative dans les textes narratifs, Paris, Grasset, 1985, 315 p.

2 Ibid., p. 102-109.

3 Ibid., p. 113-120.

4 Ibid., p. 64-86.

5 Projet aLes best-sellers au Québec, 1970-1982 n. Chercheurs principaux: Jacques Lemieux, Claude Martin, Vincent Nadeau et Denis Saint-Jacques. Subvention FCAR, 1985-1987. Voir Claude Martin, «Des best-sellers en tous genres , Cahiers de recherche sociologique, vol. IV, $\mathrm{n}^{\circ} 2$, automne 1986, p. 111-128, et $\alpha$ Comme des petits pains chauds. Essai d'économie industrielle du best-seller en français au Québec», Communication information, vol. VII, $n^{0} 3$, automne 1985, p. 107-127; aussi Denis Saint-Jacques, «La France, relais de l'américanité dans le marché du livre au Québec „, Cahiers pour la littérature populaire, automne 1987, p. 57 63, et «Ce que racontent les best-sellers *, le Risque de lire, sous la direction de Hans-Jürgen Greif, Québec, Nuit blanche, 1988, p. 113-136.

Denis Saint-Jacques, loc. cit. 
moins prévisibles du marché du livre, nous obtenons un ensemble de livres apparemment assez disparates, comportant biographies, fables et romans, d'origine tant américaine que française, allemande ou québécoise et dont les cadres de référence se situent aussi bien dans les plantations d'esclaves du Sud des ÉtatsUnis que dans le Montréal des petits restaurants ou dans la faune humaine du Banhof Zoo de Berlin-Ouest. Ces titres sont très précisément ceux que l'on retrouve sur les listes des succès de la semaine publiées dans la Presse de Montréal ou le Soleil de Québec durant les années soixante-dix et au début des années quatrevingt. Parmi les interrogations qui ont orienté nos travaux de recherche sur cette sorte de livres, il en est une toute simple: et si, en deçà des formules explicites des récits à série, se manifestait là sous des formes à peine variées une sitructure narrative commune? Si les best-sellers racontaient tous la même histoire? Il ne suffit pas en effet de le prétendre, glissant vers le préjugé et le mépris envers des œuvres prétendues fermées ${ }^{7}$; mieux vaut vérifier et arriver à définir si possible cette fabula commune. D'ailleurs, le succès qu'elle connaît sur le marché représente une appropriation nationale, une socialisation effective d'un mythe idéologique spécifique dans l'imaginaire québécois. Cette seule raison, entre autres, justifie l'attention qu'on pourrait vouloir lui prêter.

Dans les procédures de vérification et de falsification de cette hypothèse, nous avons inféré un topic de fabula hypercodée au plan idéologique ${ }^{8}$ (on rappellera en bref que le topic est un schéma hypothétique proposé par le lecteur pour reconnaître un motif, unité séquentielle, aussi bien qu'une fabula, séquence finie de macropropositions narratives) en rapport avec le lecteur statistiquement le plus probable des best-sellers: un mythe de la réussite économique de l'individu dans la société capitaliste libérale. Et cette inférence s'est avérée rentable pour l'analyse de l'ensemble de notre corpus, non sans soulever un certain nombre de problèmes que nous avons déjà abordés dans " $\mathrm{Ce}$ que racontent les best-sellers". Ce topic renvoie en fait à une fabula générale très nettement démarquée dans la majorité des récits analysés et que, faisant abstraction dans cette étude des cas problématiques, nous pouvons décrire comme scénario motif très proche de la fabula préfabriquée au sens qu'Eco donne à ces appellations ${ }^{9}$.

Avant d'en arriver à l'analyse des éléments de cette fabula, nous allons examiner brièvement les grandes caractéristiques des best-sellers en question. En premier lieu, un coup d'œil rapide sur la liste révèle la nette domination des titres d'origine américaine ou anglo-saxonne (dix sur quinze), de même que la faiblesse de la présence québécoise (réduite à un seul titre, le Matou). On constate de plus qu'un seul récit est traduit d'une autre langue que l'anglais (l'allemand, Moi, Christiane F., 13 ans, droguée, prostituée). Mais au-delà de ces

\footnotetext{
$7 \quad$ Au sens que propose $\mathrm{Eco}$, op. cit., p. 72-76.

8 Ibid., p. 109-110.

9 Ibid., 106: fabulse préfabriquées [...] schemas standards du roman policier de série, ou des groupes de fables où se répètent toujours les mêmes fonctions (au sens de Propp) dans la même succession [...] scénarios motifs, schémas assez flexibles [...] où l'on détermine certains acteurs [...], certains décors [...], certaines séquences d'actions [...], sans que pour autant soient imposées des contraintes trop précises quant à la succession des événements.
} 
différence d'origine, cette sélection de quinze best-sellers peut s'articuler en différents groupements, selon qu'on se situe au niveau des structures actorielles ou à celui des structures narratives.

Au niveau actoriel, nous distinguons d'abord les récits à protagonistes masculins (tels que le héros de Papillon ou Martin Gray dans Au nom de tous les miens, de même que Michael Corleone dans le Parrain) de ceux à personnages centraux féminins (notamment, Emma Harte dans l'Espace d'une vie, Meg Cleary dans Les oiseaux se cachent pour mourir ou les héroïnes collectives de Toilettes pour femmes). Se superposant à cette première distinction, il faut séparer les récits à protagoniste unique (Papillon, Martin Gray, Florent Boissonneault, Christiane F., Jonathan Livingston) de ceux où sont mis en scène des acteurs collectifs: soit sous la forme diachronique de lignées de personnages (Kunta Kinte et ses descendants dans Racines ou les générations successives de femmes - grandmère, mère, fille, petite-fille - dans Les oiseaux se cachent pour mourir, l'Espace d'une vie ou Tous les fleuves vont à la mer; soit encore sous l'aspect synchronique de personnages à trajectoires parallèles (les trois Jordache dans le Riche et le Pauvre, les ménagères et les féministes dans Toilettes pour femmes, ou le seigneur Toranaga et son vassal anglais Blackthorne dans Shogun).

En ce qui concerne les structures narratives et discursives, trois observations s'imposent. En premier lieu, le thème de la réussite n'est pas strictement économique, comme le suggérait notre hypothèse de départ; il subit diverses mutations qui situent la réussite sociale sur un autre plan: réalisation de soi (les Mots pour le dire), liberté et respect de la part d'autrui (Racines, Toilettes pour femmes), pouvoir et puissance (Shogun, le Parrain)... valeurs dont, dans l'imaginaire social en cause, l'avoir matériel n'est que l'équivalent général ou l'étalon de mesure, car la monnaie, sous le coup d'un tabou manifeste, ne tient pas dans la fiction ce rôle que Marx lui a reconnu pour la société concrète.

On observe également, dans les récits biographiques et dans quelques romans, que la réussite amène les protagonistes à témoigner de leur expérience par la prise de parole ou par l'écriture: Martin Gray vient témoigner au nom de tous les siens, Papillon raconte son évasion du bagne, et Jonathan Livingston prêche aux autres goélands... Comme nous le verrons ultérieurement, il s'agit là d'un thème fréquent mais non nécessaire.

Comme troisième et dernière observation, au niveau discursif en ce cas, il faut noter le souci de réalisme et d'exhaustivité dans la description des divers contextes socio-historiques de nos récits: Japon du XVII occidentale et plantations du Sud des Etats-Unis au XVIII ${ }^{\mathrm{e}}$ siècle (Racines), Australie du début du XX' de Varsovie et camp de concentration (Au nom de tous les miens)... L'importance qu'accordent ces récits à la mise en place détaillée d'un décor réaliste, explique en grande partie leur habituelle ampleur: douze des quinze récits dépassent en effet les quatre cents pages.

Ces caractéristiques permettent de construire un modèle général de ces fictions: un scénario motif, qui réunit et synthétise les aventures narratives de 
personnages de prime abord aussi différents que Kunta Kinte l'Africain, Martin Gray l'adolescent du ghetto, Florent Boissonneault, le restaurateur montréalais, Christiane F., la jeune délinquante berlinoise, Emma Harte, la fille d'employé de filature du Yorkshire devenue PDG d'une multinationale; sans oublier Toranaga le Shogun, Don Corleone, le Parrain, ainsi que tous les autres.

Partant de récits fort hétérogènes à première vue et homologués uniquement en fonction de leur succès exceptionnel sur le marché, nous aboutissons à un scénario motif de fabula cohérent et dont les isotopies peuvent à la rigueur faire penser à celui de la fabula préfabriquée. Cela mérite quelque réflexion, car il n'y a pas lieu de croire à une formule clairement identifiée par les producteurs. Toutes les recherches que l'on peut mener en ce sens interdisent de supposer que les créateurs de Jonathan Livingston le goéland, du Matou, ou de Moi, Christiane F., 13 ans, droguée, prostituée par exemple, utilisaient consciemment un modèle type de structure narrative pour la mise en forme de leurs récits respectifs. D'un autre côté, nous ne connaissons pas de contribution critique qui ait encore reconnu la présence libre de ce scénario motif dans la littérature de grande consommation. Nous insistons sur ce qualificatif, "libre», car il va de soi que dans le cas des fabulæ préfabriquées strictes et donc explicitement contraintes (policier, western, sentimental et autres séries), la recherche s'est consacrée très attentivement à cet aspect de la structure narrative bloquée, au point d'en arriver à ne plus voir beaucoup autre chose dans ces textes ${ }^{10}$. Toutefois, s'agissant de récits de grande consommation non spécifiquement classés, comme ceux de notre corpus, personne ne semble vraiment s'être donné la peine de décrire une intrigue errante ${ }^{11}$ pourtant facile à pressentir. Cette "liberté " veut dire entre autres que les contraintes de la fabula que nous avons reconnue ne sont pas obligatoires pour la classe considérée; même si la plupart s'y plient, il y a de fait des best-sellers qui ne s'y conforment pas, voir par exemple Love Story d'Erich Segal (Paris, Montaigne, 1972). Mais cela marque également que les contraintes en question résultent d'une détermination inaperçue par les agents qui y sont soumis dans un de ces effets de captation imaginaire caractéristique de l'assujettissement idéologique. On peut présumer qu'en pareil cas, le scénario motif en tant que topic pragmatique et la fabula type en tant qu'isotopie sémantique vont tendre à se confondre. À vrai dire, le scénario ne pouvant être déduit qu'à posteriori, une telle supposition ne pourrait rencontrer de vérification expérimentale effective. Il est permis de croire en tout cas que producteurs et consommateurs ont les mêmes rapports au topic fondés sur l'habitus ${ }^{12}$, d'où une manifestation concrète plus souple que dans le cas des fabulæ préfabriquées; en effet, les déterminations sociohistoriques changeantes ne rencontrent pas en ce cas d'entrave explicite aux manipulations qu'elles font subir à une structure narrative à peine reconnue.

10 Voir Julia Bettinotti, la Corrida de l'amour. Le roman Harlequin, Montréal, Université du Québec à Montréal, 1986, 160 p. ou Charles Grivel, «Observations du roman policier *, Entretiens sur la paralittérature, Paris, Plon, 1970, p. 229-258, exemple extrême et caricatural de déduction systématique où l'auteur se scandalise de la pauvreté de ce qu'il vient de dépouiller.

11 Tomachevsky 1928, voir Umberto Eco, op. cit., p. 105.

12 Voir Pierre Bourdieu, le Sens pratique, Paris, Minuit, 1980, p. 88 et suivantes. 
Reprenons la description, motif par motif, de notre fabula: 1 - un personnage vit dans une situation d'équilibre euphorique; 2 - apparaissent des difficultés graves mettant en jeu sa survie ou sa liberté; 3 - le protagoniste résiste à l'adversité et se prépare avec détermination à renverser la situation (c'est normalement la phase longue du récit); 4 -il subit un échec dans l'ordre affectif; 5-il parvient au succès; 6 - il connaît un rétablissement dans l'ordre affectif; 7 - il prend la parole pour narrer sa propre aventure. Disons immédiatement que seuls les quatre motifs centraux (de 2 à 5) sont nécessaires à la réalisation de la fabula; ils apparaissent ainsi sinon théoriquement indissociables, du moins historiquement toujours coprésents, formant en pratique un motif unique. On notera que le cinquième et le sixième, ceux de la réussite, peuvent n'apparaître que comme horizon à la fin du récit, tout à fait comme le mariage dans la comédie classique, thème obligé, mais habituellement réduit à l'état de suite virtuelle assurée. Ici et là, les tribulations occupent toute la place et les mariages heureux n'ont pas d'histoire. Voilà de toute façon les quatre motifs stables de notre fabula dans leur succession la plus habituelle: adversité, résistance, échec affectif et victoire sur l'adversité. L'ordre logique de succession du deuxième, du troisième et du cinquième s'avère impossible à modifier (dégradation, résistance et renversement); il n'en va pas de même pour le quatrième (échec affectif) qui peut précéder, coïncider avec ou suivre le renversement. Disons simplement qu'il lui est lié syntaxiquement et donc l'accompagne d'assez près en satellite. Ce motif suffirait à lui seul à faire réévaluer la suffisance critique qui tend à décrire les best-sellers comme des histoires simplistes où tout finit bien. Et cette unité thématique se révèle d'autant plus intéressante à étudier que le plus fréquemment, la trame discursive du récit ne motive d'aucune façon la nécessité de sa présence. En quoi la mort de Monsieur Émile s'impose-t-elle dans le Matou ? Pourquoi faut-il que Martin Gray perde une deuxième fois sa famille dans Au nom de tous les miens? À quoi sert le mensonge par lequel le nouveau Don Corleone trompe sa femme à la fin du Parrain? Ces contraintes de la fabula commune apparaissent au plan de la manifestation comme aléatoires, mais la redondance du motif et sa position répondent: la réussite est à ce prix. Nous ne sommes ici pas si loin de l'hubris tragique, même si les déséquilibres de la structure narrative connaissent en ce cas une évolution d'un genre différent.

Le premier motif variable, celui de l'état antérieur euphorique, n'a que ceci de remarquable: le fait d'être aléatoire. En effet, certains de nos récits commencent par la deuxième phase de la fabula, celle de la dégradation. La situation malheureuse est alors présentée non en référence à un état antérieur plus satisfaisant, mais plutôt en rapport avec d'autres situations de personnages concurrents privilégiés. Il en est ainsi par exemple du Riche et le Pauvre où la vie de famille malheureuse à partir de laquelle s'enclenche la fabula sert en fait de repoussoir aux héros dans leur quête de réussite. Notons encore que le motif du rétablissement affectif est très incertain, aussi souvent présent qu'absent. L'ultime motif variable, celui de la prise de parole, fonctionne lui aussi de façon aléatoire; on le retrouve de façon particulièrement marquée dans Au nom de tous les miens, dans Jonathan Livingston le goéland, dans Racines et dans les Mots pour le dire. Il nous permet de saisir que, même dans la littérature de 
grande consommation, il est un effet réflexif de l'acte narratif sur les thèmes de l'histoire ou de la fabula représentée. L'autoreprésentation du geste narratif luimême n'est pas exclusive à la grande littérature.

La présence de ce scénario motif de la réussite, de même que la diversité et la complexité des univers narratifs dans lesquels il s'inscrit, suggèrent en somme un lecteur modèle $e^{13}$ dont l'encyclopédie ${ }^{14}$ comprendrait, au niveau des scénarios et de l'hypercodage idéologique, une sensibilité au topic de la réussite sociale, un souci de vraisemblance socio-historique, ainsi qu'un certain relativisme libéral en ce qui concerne la façon d'envisager les univers narratifs.

De fait, ces caractéristiques du lecteur modèle des best-sellers correspondent effectivement aux tendances centrales d'une enquête menée au début des années 1980 par Sylvie Provost, auprès de lecteurs et lectrices francophones de Montréal ${ }^{15}$. Les répondants de cette enquête ont confirmé la validité de notre échantillon de best-sellers. On retrouve ainsi en tête de la liste des ouvrages qui ont le plus impressionné ces lecteurs: Les oiseaux se cachent pour mourir, l'Espace d'une vie et Toilettes pour femmes ${ }^{16}$. Ils souhaitent en effet que leurs lectures de loisir soient non seulement distrayantes, mais aussi enrichissantes: $55,8 \%$ des répondants souhaitent être transportés dans un univers différent du leur, $50,3 \%$ désirent y voir représentés des personnages comportant des traits de caractère ou une personnalité qu'ils admirent ${ }^{17}$; en même temps, $78 \%$ espèrent que l'cuvre offre des idées auxquelles ils n'avaient jamais pensé; enfin, pour $85 \%$ des lecteurs et lectrices, l'œuvre doit aussi présenter une peinture du réel ${ }^{18}$.

Pour un lecteur typique, lire des best-sellers ne sert pas seulement à passer agréablement ses moments de détente: il croit aussi y apprendre des choses nouvelles sur la Deuxième Guerre mondiale et l'antisémitisme, sur le crime organisé, la délinquance, le féminisme, le mouvement de libération des Noirs, le fondamentalisme musulman, le terrorisme ou la culture de ces Japonais devenus si puissants... en somme, ce qui donne de la consistance socio-historique aux grands titres de son quotidien ou aux images de son téléviseur.

Si le lecteur modèle prévu par le best-seller était conforme à ce «lecteur concret moyen" des enquêtes, on pourrait s'attendre à ce que le récit à succès soit un exemple typique de texte fermé, où le soin accordé au détail réaliste consiste en une opération de fermeture. Mais il faut comprendre que la notion de "lecteur concret moyen " désigne non pas des catégories spécifiques de lecteurs, mais plutôt un dénominateur commun aux diverses couches sociales qui forment ce vaste conglomérat aux contours indéfinis que désigne la notion de "classes moyennes".

13 Umberto Eco, op. cit., p. 64-86.

14 Ibid., p. 17-32.

15 Sylvie Provost, a La lecture de loisir des Montréalais et Montréalaises d'instruction moyenne », thèse de doctorat en littérature québécoise, Québec, université Laval, 1987, 308 f.

16 Ibid, p. 98. Il faut tenir compte du moment de l'enquête, année 1981, pour expliquer la présence de ces titres plutôt que d'autres beaucoup plus antérieurs ou postérieurs dans l'échantillon.

17 Ibid., p. 49.

18 Ibid., p. 92. 
Le lecteur de best-sellers, c'est à peu près n'importe qui, hormis les analphabètes ou l'avant-garde littéraire. Toutefois, le profil-type du lecteur de best-sellers démontre une "tendance lourde", en ce sens que le lecteur est la plupart du temps une lectrice ${ }^{19}$, et que la minorité d'hommes lecteurs de bestsellers révèle une sélectivité plus restreinte dans son choix de lectures.

En effet, à peine 5\% des hommes interrogés par Sylvie Provost admettent trouver de l'intérêt dans des écrits féministes tels que Toilettes pour femmes ou les Mots pour le dire, et aucun n'affirme avoir été impressionné par des récits à protagonistes féminines comme l'Espace d'une vie ou les Oiseaux se cachent pour mourir 20 . Par contre, les lectrices qui déclarent se passionner avant tout pour des récits d'action, que nos hypothèses de départ caractérisaient en "bestsellers pour hommes" (Shogun, le Cinquième Cavalier), se retrouvent dans les mêmes proportions (17\%) que les répondants de sexe masculin ${ }^{21}$.

Cuvres fermées, les best-sellers? On peut en douter. Certes, une lecture raciste de Racines ou de Au nom de tous les miens, une lecture antiféministe de Toilettes pour femmes, relèveraient davantage de "l'utilisation" que de "l'interprétation" de ces textes ${ }^{22}$. Mais que dire de Jonathan Livingston le goéland? Quelle est l'interprétation exacte de cette courte fable (87 p.), située dans un univers si loin du réalisme, et où pourtant se réalisent pleinement les six motifs de notre scénario? À notre avis, l'ouverture de ce texte est précisément liée à sa pauvreté référentielle et au côté "merveilleux" de l'univers narratif: le vague de la fable permet d'envisager diverses formes du motif de la réussite.

L'ensemble des quinze titres comporte aussi, parmi les récits longs au contexte réaliste, d'autres œuvres qu'on pourrait qualifier d'ouvertes. Ainsi, dans le Riche et le Pauvre, les trois Jordache présentent trois trajectoires bien différentes: Rudy réussit en affaires, mais échoue en amour et en politique; Gretchen connaît aussi divers déboires affectifs et professionnels, puis retrouve son équilibre grâce à un emploi assez modeste dans l'industrie cinématographique; enfin Tom, le mouton noir, finit par se ranger, réalise son rêve d'acquérir un yacht, mais est assassiné pour avoir tíré la femme de Rudy des griffes d'un truand. Laquelle de ces trajectoires peut être considérée comme exemplaire? C'est une question d'interprétation. De même, que dire de l'accession de Michael Corleone à la tête de la Mafia? En quoi ce grand féodal, qui, pour accéder au pouvoir, utilise la ruse et la violence, est-il plus immoral que cet autre grand seigneur, Toranaga le Shogun? Et en quoi Barzini et Ishido, antagonistes du Parrain et du Shogun, sont-ils différents des protagonistes, hormis le fait qu'ils échouent?

De fait, qu'importe la réussite de Don Corleone, de Toranaga ou de chacune des féministes de Toilettes pour femmes? Puisque le topic de la réussite

19 Selon des chiffres du ministère dés Affaires culturelles du Québec, 61\% des Québécois lecteurs de livres sont de sexe féminin (M.A.C.', Chiffres à l'appui, vol. II, $n^{\circ} 1$, avril 1984, p. 1); de même les femmes représentent 66,5\% des 170 répondants de l'enquête de Sylvie Provost.

20 Sylvie Provost, op. cit., p. 107.

21 Ibid., p. 71.

22 Umberto Eco, op. cit., p. 76. 
demeure constant, l'information se trouve en fait dans le cadre proposé: la Mafia, le Japon, le féminisme.

En somme, nous émettons l'hypothèse que le circuit restreint de la littérature n'a pas le monopole de l'œuvre ouverte. Affirmer le contraire serait faire preuve d'une conception qui mériterait une sérieuse critique sociologique: pourquoi les seuls produits culturels "ouverts" seraient-ils ceux qui intéressent les "intellectuels "? À notre avis, l'ouverture ou la fermeture d'un texte ne relève pas seulement de ses isotopies sémantiques, mais aussi de l'environnement idéologique du lecteur concret. Si le lecteur modèle que présuppose le best-seller se situe au centre social et idéologique des sociétés industrialisées, il y a nécessairement une variance autour de ce centre, et par conséquent une ouverture probable de ces œuvres de grande consommation.

Aussi considérons-nous qu'une des tâches prioritaires des chercheurs en littérature, en particulier de ceux qui explorent la littérature de masse, consiste à développer des analyses plus fines du public lecteur.

En effet, le recours aux enquêtes auprès de grands échantillons de lectrices et de lecteurs (telle que celle de Sylvie Provost ou celles commanditées par le ministère des Affaires culturelles) apporte des informations déjà très utiles, mais dont les limites s'avèrent gênantes. Et cela, d'une part, parce que les hasards de l'échantillonnage produisent peu de répondants ayant effectivement lu un nombre suffisant de titres qui intéressent les chercheurs, d'autre part, parce que la méthode du questionnaire ne permet pas une analyse en profondeur des attentes et des motivations des répondants. Il faut par conséquent envisager des analyses plus qualitatives, impliquant des groupes de lecture spécifiquement constitués pour l'analyse de la "réception publique " d'œuvres préalablement identifiées. Nous envisageons du reste la poursuite de nos recherches dans cette perspective.

Nous croyons qu'une telle approche permettrait une confrontation fort utile, entre d'une part les exégèses savantes et les lecteurs modèles théoriques, et d'autre part les usages sociaux réels que tirent de leurs lectures les lectrices et lecteurs concrets.

\section{Annexe}

CHARRIÈRRE, Henri, Papillon, Paris, Laffont, 1969, 520 p. Français, biographie: évasion réussie du bagne de Cayenne.

PUZO, Mario, le Parrain, Paris, Laffont, 1970, 488 p. Américain, roman: l'accession au pouvoir d'un chef de la Mafia.

GRAY, Martin, Au nom de tous les miens, Paris, Laffont, 1971, 416 p. Français, biographie: un Juif polonais survit aux camps de concentration, fait fortune, trouve le bonheur et perd sa famille.

BACH, Richard, Jonathan Livingston le goéland, Paris, Flammarion, 1973, 87 p. Américain, fable: un goéland poursuit la perfection du vol et tente d'y convertir ses semblables.

CARDINAL, Marie, les Mots pour le dire, Paris, Grasset et Fasquelle, 1975, 345 p. Français, littéraire, biographie: une femme raconte sa psychanalyse. 
CLAVELL, James, Shogun, Montréal, Libre Expression, 1977, 976 p. Américain, roman: au XVI' siècle, un seigneur en extrême difficulté renverse la situation et devient Shogun.

HALEY, Alex, Racines, Paris, Alta, 1977, 476 p. Américain, chronique: une famille noire de génération en génération, de l'Afrique aux États-Unis par l'esclavage et la libération. Enfin, le retour aux sources.

SHAW, Irwin, le Riche et le Pauvre, Paris, Presses de la Cité, 1977, 513 p. Américain, roman: une famille d'immigrés allemands réussit aux Etats-Unis.

McCULLOUGH, Colleen, Les oiseaux se cachent pour mourir, Paris, Belfond, 1978, 536 p. Américain, roman: la mère, la fille et la petite-fille face à l'amour en Australie. Une liaison avec un cardinal.

FRENCH, Marilyn, Toilettes pour femmes, Paris, Laffont, 1979, 488 p. Américain, roman: chronique d'une libération féministe.

TAYLOR-BRADFORD, Barbara, l'Espace d'une vie, Paris, Belfond, 1980, 559 p. Américain, roman: une pauvre fille trompée fait fortune et se venge.

LAPIERRE, Dominique et Larry COLLINS, le Cinquième Cavalier, Paris, Laffont, 1980, 461 p. Américano-français, roman: politique fiction. Khadafi prend Manhattan en otage.

BEAUCHEMIN, Yves, le Matou, Montréal, Québec-Amérique, 1981, 582 p. Québécois, littéraire, roman: un Québécois s'établit restaurateur.

HERMANN, Kai et Horst RIECK, Moi Christiane F., 13 ans, droguée, prostituée, Paris, Mercure de France, 1981, 279 p. Allemand, biographie: le titre est clair.

PLAIN, Belva, Tous les fleuves vont à la mer, Paris, Presses de la Renaissance, 1982, 523 p. Américain, roman: une passion secrète et l'immigration juive aux États-Unis. 